\title{
Robustness of Greedy Type Minimum Evolution Algorithms
}

\author{
Takeya Shigezumi \\ c/o Prof. O. Watanabe, \\ Dept. of Mathematical and Computing Sciences, \\ Tokyo Institute of Technology \\ Takeya.Shigezumi@is.titech.ac.jp
}

\begin{abstract}
For a phylogeny reconstruction problem, Desper and Gascuel 2 proposed Greedy Minimum Evolution algorithm (in short, GME) and Balanced Minimum Evolution algorithm (in short, BME). Both of them are faster than the current major algorithm, Neighbor Joining (in short, NJ); however, less accurate when an input distance matrix has errors. In this paper, we prove that BME has the same optimal robustness to such errors as NJ but GME does not. Precisely, we prove that if the maximum distance error is less than a half of the minimum edge length of the target tree, then BME reconstruct it correctly. On the other hand, there is some distance matrix such that maximum distance error is less than $\frac{2}{\sqrt{n}}$ of the minimum edge length of the target tree, for which GME fails to reconstruct the target tree.
\end{abstract}

\section{Introduction}

A phylogeny reconstruction problem is to determine an evolutionary tree representing relationships between a set of species from a distance matrix expressing the "closeness" of each pair of species. Recently, the phylogeny reconstruction is not only used for biology, but also for clustering various kinds of data, such as languages 10, music, etc. 11] To state the phylogeny construction problem formally, we need some notations. For any $n$, we use $L=\{1, \ldots, n\}$ to denote a set of $n$ species. A distance matrix $D=\left(d_{i j}\right)$ over $L$, for each pair $i$ and $j$ in $L$, a distance $d_{i j} \geq 0$. Note that $d_{i j}=d_{j i}$ and that $d_{i i}=0$. An evolutionary tree over $L$ is a tree such that its set of leaves is $L$ and all internal vertices are of degree three. For any tree $T$ and for any $i$ and $j$ in $L$, let $d_{i j}^{T}$ denote the length of the unique path between $i$ and $j$, i.e., the sum of the length of edges on the path. Now the phylogeny construction problem is stated as follows.

Instance: A distance matrix $D=\left(d_{i j}\right)$ over $L=\{1, \ldots, n\}$.

Question: Find a tree $T^{*}$ minimizing the following square error:

$$
\sum_{i<j}\left|d_{i j}-d_{i j}^{T^{*}}\right|^{2}
$$


Day [8] showed that this problem is NP-hard in general. It is, however, possible to "reconstruct" a tree in polynomial time if a distance matrix is sufficiently close enough to the one for a given tree. Note that for any tree $T$, this $T$ itself is the unique solution for the phylogeny construction problem on the distance matrix $D^{T}=\left(d_{i j}^{T}\right)$. Several polynomial time algorithms have been proposed for reconstructing the original tree $T$ from $D^{T}$. Among them, Neighbor-Joining [4, its variants BIONJ [5] and WEIGHBOR 6], and Fitch-Margoliash as implemented by Felsenstein [9] are well known. NJ and its variants run in $O\left(n^{3}\right)$ for reconstructing a tree of $n$ leaves. Though polynomial, this running time is not sufficient enough for constructing a large evolutionary trees, and several attempts have been made to improve the efficiency .

Recently, Desper and Gascuel [2] introduced Greedy Minimum Evolution (GME) algorithm based on the ordinary least squares (OLS) branch length estimation and Balanced Minimum Evolution (BME) algorithm based on a branch length estimation scheme of Pauplin 3. BME runs in $O\left(n^{2} \operatorname{diam}(T)\right)$ where $\operatorname{diam}(T)$ is a diameter of the target tree, and GME runs in $O\left(n^{2}\right)$, which is optimal considering that the size of the input distance matrix is $O\left(n^{2}\right)$. Moreover, BME has an important property that the output tree has no edge with negative length [1. Since edges with negative length are biologically meaningless, this property is also an advantage of BME.

In practice, a distance matrix has errors, that is, some distance $\delta_{i j}$ differs from the original distance $d_{i j}^{T}$. For measuring such errors, Atteson [7] introduced the notation of " $l_{\infty}$ radius." We say that a distance matrix $\Delta=\left(\delta_{i j}\right)$ has $l_{\infty}$ radius $\alpha$ to an original distance $D^{T}=\left(d_{i j}^{T}\right)$ if

$$
\left\|D^{T}-\Delta\right\|_{\infty}=\max _{i<j}\left|d_{i j}^{T}-\delta_{i j}\right|<\alpha \min _{e \in E(T)}\{l(e)\},
$$

where $E(T)$ is the set of edges of $T$ and $l(e)$ denotes the length of an edge $e$. An algorithm has $l_{\infty}$ radius $\alpha$ if it yields the original $T$ for any distance matrix of $l_{\infty}$ radius $\alpha$ to $D^{T}$. Note that no algorithm has $l_{\infty}$ radius larger than $\frac{1}{2}$. Because if we allow errors larger than $\frac{1}{2} l(e)$, there exists one distance matrix made from two different trees with such errors. Atteson showed that NJ has $l_{\infty}$ radius $\frac{1}{2}$.

Desper and Gascuel showed the relationship between BME length estimation and weighted least squares branch length estimation 1 as a theoretical foundation of BME. However, there is still no theoretical analysis about $l_{\infty}$ radius for GME and BME. We analyze BME and GME, and obtain the following two theorems, which claim that BME has $l_{\infty}$ radius $\frac{1}{2}$ but GME does not.

Theorem 1. BME has $l_{\infty}$ radius $\frac{1}{2}$, i.e., if the input distance matrix $\Delta$ satisfies

$$
\left\|D^{T^{*}}-\Delta\right\|_{\infty}<\frac{1}{2} \min _{e \in E\left(T^{*}\right)}\{l(e)\}
$$

BME outputs $T^{*}$.

Theorem 2. Let $\alpha_{G}$ be an $l_{\infty}$ radius of GME. Then,

$$
\alpha_{G}<\frac{2}{\sqrt{n}}
$$


According to these theorems, BME has the same robustness as NJ, and it is faster than NJ. On the other hand, GME is faster than BME, it is less accurate in some cases. Our proof is obtained by a precise analysis of the proof of Desper and Gascuel 1 for the correctness of BME on error free distance matrix. Desper and Gascuel also proposed Balanced Nearest Neighborhood Interchange (BNNI) algorithm to improve a phylogeny tree into minimal one. However, according to our theorem, we do not need to use BNNI when BME is used for a distance matrix $\Delta$ with sufficiently small $\left\|D^{T^{*}}-\Delta\right\|_{\infty}$ error.

\section{Preliminaries}

We call $T^{*}$ the target tree being studied with an edge set $E\left(T^{*}\right)$ and edge weight function $l: E\left(T^{*}\right) \rightarrow \mathbb{R}$. Let $l_{\text {min }}^{T^{*}}=\min _{e \in E\left(T^{*}\right)}\{l(e)\}$ and $\Delta=\left(\delta_{i j}\right)$ be an estimate of the evolutionary distance between leaves $i$ and $j$. Let $E=\left(\varepsilon_{i j}\right)$ be an error matrix given by following equation:

$$
\varepsilon_{i j}=\delta_{i j}-d_{i j}^{T^{*}}
$$

and $\varepsilon_{0}$ be the maximum error such that $\varepsilon_{0}=\max _{i, j}\left|\varepsilon_{i j}\right|$. For a given tree $T$, we say $P_{i j}^{T}$ be a number of edges on the path connecting $i$ and $j$ on the tree $T$, and say $r(A)$ for any subtrees $A$ of $T$ be a root vertex of the subtree. Let $P_{A B}^{T}$ be a number of edges from $r(A)$ to $r(B)$ for any disjoint subtrees $A$ and $B$. We will treat a subtree $A$ as a set of leaves for a simpler notation.

Pauplin [3] showed BME principle to estimate the edge length by modifying the OLS analytical formulae. Under this principle, the tree length is estimated as $\hat{l}(T, \Delta)=\sum_{i, j} 2^{1-P_{i j}^{T}} \delta_{i j}$. Consider the tree $T$ in Figure 1, where $k$ is inserted between subtrees $C$ and $A \cup B$, and assume that we have the size $\hat{l}(T, \Delta)$ of this new tree. If we know the size of $T$, we can quickly calculate the size of $T^{\prime}$ using the following equation:

$$
\hat{l}\left(T^{\prime}, \Delta\right)=\hat{l}(T, \Delta)+\frac{1}{4}\left\{\left(\delta_{A C}^{T}+\delta_{k B}^{T}\right)-\left(\delta_{A B}^{T}+\delta_{k C}^{T}\right)\right\} .
$$

In this formula, $\delta_{A B}^{T}$ is defined as follows. If $A$ and $B$ each contains only one leaf, denoted as $a$ and $b$, respectively, then $\delta_{A B}^{T}=\delta_{a b}$, while if one of the two, say B, is made of two subtrees denoted as $B_{1}$ and $B_{2}$, then $\delta_{A B}^{T}=\frac{1}{2}\left(\delta_{A B_{1}}^{T}+\delta_{A B_{2}}^{T}\right)$.

Under the OLS length estimation principle, (1) is represented as follows:

$$
\begin{aligned}
\hat{l}\left(T^{\prime}, \Delta\right)-\hat{l}(T, \Delta)= & \frac{1}{2}\left[\left(\lambda-\lambda^{\prime}\right)\left(\Delta_{k \mid A}+\Delta_{B \mid C}\right)\right. \\
& \left.+\left(\lambda^{\prime}-1\right)\left(\Delta_{A \mid B}+\Delta_{k \mid C}\right)+(1-\lambda)\left(\Delta_{A \mid C}+\Delta_{k \mid B}\right)\right]
\end{aligned}
$$

where $\lambda=\frac{(|A|+|B||C|)}{(|A|+|B|)(|C|+1)}, \lambda^{\prime}=\frac{(|A|+|B||C|)}{(|A|+|C|)(|B|+1)}$ and $\Delta_{A \mid B}=\frac{1}{|A||B|} \sum_{i \in A, j \in B} \delta_{i j}$.

We here introduce BME algorithm by Desper and Gascuel [2]. This algorithm starts with the tree of three leaves and adds the leaf to minimize the estimated tree length. 

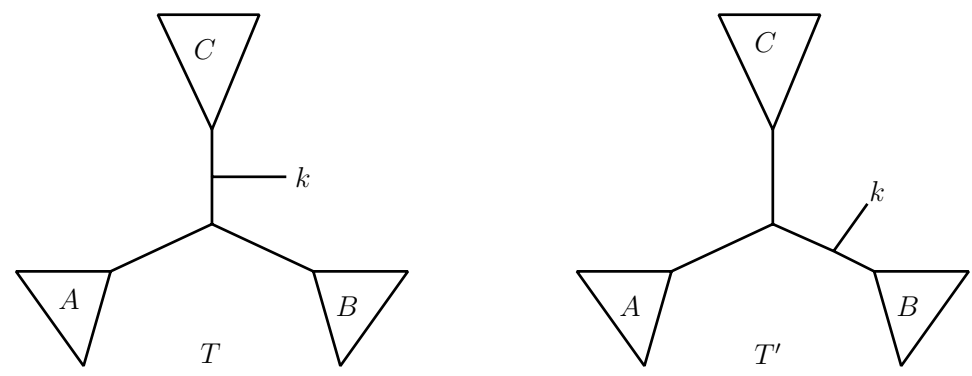

Fig. 1. Positions the leaf $k$ is inserted to

BME algorithm is as follows:

- For $k=3$, initialize the matrix of balanced average distances between distant- 2 subtrees and the array counting the number of leaves par subtree. Form $T_{3}$ with leaf set $\{1,2,3\}$.

- For $k=4$ to $n$,

1. Compute all $\delta_{k S}^{T_{k-1}}$ balanced average distances;

2. Starting from an initial edge $e_{0}$ of $T_{k-1}$, set $f\left(e_{0}\right)=0$, and recursively search each edge $e$ to obtain $f(e)$ from (1).

3. Select the best edge by minimizing $f$, insert $k$ on the edge to form $T_{k}$, and update the balanced average distance between every pair of distant-2 subtrees as well as the number of leaves per subtree.

$-\operatorname{Return} T_{n}$.

The time complexity of BME is $O\left(n^{2} \operatorname{diam}\left(T^{*}\right)\right)$. GME is almost same but using (2) and average distance $\Delta_{A \mid B}$ instead of the equation 1 and $\delta_{A B}^{T}$. The time complexity of GME is $O\left(n^{2}\right)$.

\section{Robustness of BME}

BME algorithm chooses the position of the leaf $k$ step by step. Let $T_{k}^{*}$ be a tree over $\{1, \ldots, k\}$ induced from the target tree $T^{*}$. The proof of Theorem 1 is by the induction of $k$. In $k=3$, there is only one topology $T_{3}^{*}$. Let us consider the $k$-th round. By the induction hypothesis, BME reconstructs the target tree topology $T_{k-1}^{*}$. BME chooses $T_{k}$ minimizing $\hat{l}\left(T_{k}, \Delta_{k}\right)$, where $\Delta_{k}$ is a distance matrix on $\{1,2, \ldots, k\}$ induced from $\Delta$.

For any $T_{k}^{\prime} \neq T_{k}^{*}$, we prove the following inequality holds if $\varepsilon_{0}<\frac{1}{2} l_{\min }^{T^{*}}$.

$$
\begin{aligned}
\hat{l}\left(T_{k}^{\prime}, \Delta_{k}\right)- & \hat{l}\left(T_{k}^{*}, \Delta_{k}\right) \\
& =\sum_{i j} 2^{1-P_{i j}^{T_{k}^{\prime}}} \varepsilon_{i j}-\sum_{i j} 2^{1-P_{i j}^{T_{k}^{*}}} \varepsilon_{i j}+\hat{l}\left(T_{k}^{\prime}, D_{k}^{T^{*}}\right)-\hat{l}\left(T_{k}^{*}, D_{k}^{T^{*}}\right)>0 .
\end{aligned}
$$



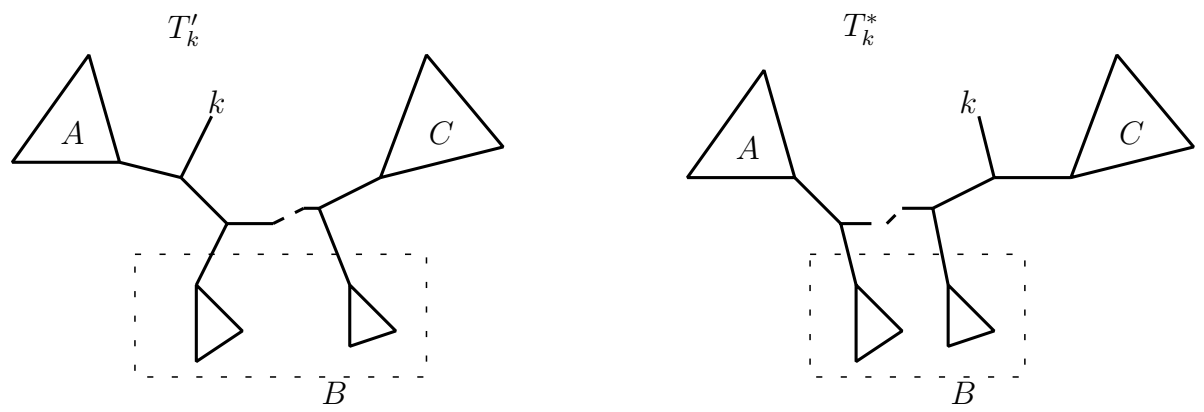

Fig. 2. $T_{k}^{*}$ and $T_{k}^{\prime}$

We call the value of the first two terms of the last equation the error difference and the value of the last two terms the tree difference.

We here provide two lemmas about the error difference and tree difference. Their precise proofs are shown in 12. Let $T_{k}^{\prime}$ and $T_{k}^{*}$, and name subtrees $A, B$ and $C$ of $T_{k-1}$ as shown in Figure 2, Let $p=P_{A C}^{T_{k}-1}$, i.e., number of edges on the path between the root of $A$ and the root of $C$ of $T_{k-1}$.

\section{Error Difference}

\section{Lemma 1}

$$
\begin{aligned}
\sum_{i j} 2^{1-P_{i j}^{T_{k}^{\prime}}} \varepsilon_{i j}-\sum_{i j} 2^{1-P_{i j}^{T_{k}^{*}}} \varepsilon_{i j} & \\
& \geq-3 \varepsilon_{0}+2 \cdot 2^{-p} \varepsilon_{0}+ \begin{cases}3 \cdot 2^{-\frac{p}{2}} \varepsilon_{0} & (p: \text { even }) \\
2 \cdot 2^{-\left\lfloor\frac{p}{2}\right\rfloor} \varepsilon_{0} & (p: \text { odd })\end{cases}
\end{aligned}
$$

\section{Tree Difference}

\section{Lemma 2}

$$
\hat{l}\left(T_{k}^{\prime}, D_{k}^{T^{*}}\right)-\hat{l}\left(T_{k}^{*}, D_{k}^{T^{*}}\right) \geq l_{\text {min }}^{T_{k}^{*}}\left(p-2+2 \cdot 2^{-p}\right) .
$$

\section{Proof of the Theorem 1}

Now, we obtained that for any topology $T_{k}^{\prime} \neq T_{k}^{*}$, the difference of the estimated tree length is

$$
\begin{aligned}
& \hat{l}\left(T_{k}^{\prime}, \Delta_{k}\right)-\hat{l}\left(T_{k}^{*}, \Delta_{k}\right) \\
& \quad \geq l_{\text {min }}^{T_{k}^{*}}\left(p-2+2 \cdot 2^{-p}\right)-3 \varepsilon_{0}+2 \cdot 2^{-p} \varepsilon_{0}+ \begin{cases}3 \cdot 2^{-\frac{p}{2}} \varepsilon_{0} & (p: \text { even }) \\
2 \cdot 2^{-\left\lfloor\frac{p}{2}\right\rfloor} \varepsilon_{0} & (p: \text { odd })\end{cases}
\end{aligned}
$$

Under the condition $\varepsilon_{0}=\left\|D^{T^{*}}-\Delta\right\|_{\infty}<\frac{1}{2} l_{\text {min }}^{T^{*}} \leq \frac{1}{2} l_{\text {min }}^{T_{k}^{*}}$, the estimated difference is larger than 0 for any $p \geq 2$. Note that if $p=1$, then $T_{k}^{*}=T_{k}^{\prime}$. 


\section{Robustness of GME}

We consider a special case of input to prove the theorem 2. If $\varepsilon_{0}$ exceeds $\alpha_{G}$, there is an input for which GME outputs a wrong tree. Same as the proof of theorem 1, let $T_{k}^{*}$ be a target tree in $k$-th round of GME, composed of subtrees $A, B, C$ and a leaf $k$. Let $T_{k}^{\prime}$ be a tree obtained from $T_{k}^{*}$ by swapping subtree $A$ and leaf $k$. GME uses the following equation to compare the estimated tree lengths:

$$
\begin{aligned}
\hat{l}\left(T_{k}^{\prime}, \Delta_{k}\right)-\hat{l}\left(T_{k}^{*}, \Delta_{k}\right)= & \frac{1}{2}\left[\left(\lambda-\lambda^{\prime}\right)\left(\Delta_{k \mid A}+\Delta_{B \mid C}\right)\right. \\
& \left.+\left(\lambda^{\prime}-1\right)\left(\Delta_{A \mid B}+\Delta_{k \mid C}\right)+(1-\lambda)\left(\Delta_{A \mid C}+\Delta_{k \mid B}\right)\right],
\end{aligned}
$$

where $\lambda=\frac{(|A|+|B||C|)}{(|A|+|B|)(|C|+1)}$ and $\lambda^{\prime}=\frac{(|A|+|B||C|)}{(|A|+|C|)(|B|+1)}$.

We obtained the following lemma by the same way as the theorem 1 .

Lemma 3. Let $e$ be an edge on $T_{k}^{*}$ separating $A$ and $B$ from $C$ and $k$.

$$
\hat{l}\left(T_{k}^{\prime}, \Delta_{k}\right)-\hat{l}\left(T_{k}^{*}, \Delta_{k}\right)=\left(1-\lambda^{\prime}\right) l^{T_{k}^{*}}(e)- \begin{cases}2\left(1-\lambda^{\prime}\right) \varepsilon_{0} & (|B| \geq|C|) \\ 2(1-\lambda) \varepsilon_{0} & (|B|<|C|)\end{cases}
$$

Proof. See [12].

If $|B| \geq \mid C$ and $\varepsilon_{0}<\frac{1}{2} l^{T_{k}^{*}}(e)$, GME chooses $T_{k}^{*}$. However, for $A, B, C$ and $\varepsilon_{0}$ satisfying $|B|<|C|$ and $\varepsilon_{0}>\frac{\left(1-\lambda^{\prime}\right)}{2(1-\lambda)} l^{T_{k}^{*}}(e)$, GME chooses $T_{k}^{\prime}$. Consider when the leaf $k$ is the last leaf. in this case, FASTNNI after GME cannot correct this fault.

Let $F(a, b, c)$ be:

$$
F(a, b, c)=\frac{\left(1-\lambda^{\prime}\right)}{2(1-\lambda)}=\frac{(a+b)(c+1)(a b+c)}{(a+c)(b+1)(a c+b)}
$$

where $a, b$ and $c$ are the cardinality of $A, B$ and $C$ respectively. By the definition of $\alpha_{G}$, if $\varepsilon_{0}<\alpha_{G} l_{\text {min }}^{T^{*}}$ then GME succeeds to output $T^{*}$, we obtained $\alpha_{G} \leq$ $\min _{b<c, a+b+c+1=n} F(a, b, c)$. We observed that $F(a, b, c)$ is minimized when $a=$ $\sqrt{n}, b=\sqrt{n}-4$ and $c=n-2 \sqrt{n}+3$. Now the theorem is obtained:

$$
\begin{aligned}
\alpha_{G} \leq \min _{\substack{0<a, 0<b<c, a+b+c+1=n}} F(a, b, c) \leq F(\sqrt{n}, \sqrt{n}-4, n-2 \sqrt{n}+3) & \\
& =\frac{2}{\sqrt{n}}-\frac{2}{n}-\frac{5}{n \sqrt{n}}+\frac{12}{n^{2}}+\ldots<\frac{2}{\sqrt{n}} .
\end{aligned}
$$

\section{Concluding Remarks}

We proved BME has $l_{\infty}$ radius $\frac{1}{2}$ and GME has $l_{\infty}$ radius less than $\frac{2}{\sqrt{n}}$. Desper and Gascuel presented BNNI and FASTNNI to modify an initial tree to the 
minimal one. However, if $\varepsilon_{0}<\frac{1}{2} \min _{e \in E\left(T^{*}\right)} l(e)$, we does not need to use BNNI. If $\varepsilon_{0}>\frac{1-\lambda^{\prime}}{2(1-\lambda)} \min _{e \in E\left(T^{*}\right)} l(e)$, and GME fails to put the last leaf, FASTNNI cannot correct such fault.

\section{References}

1. R. Desper, O. Gascuel. Theoretical Foundation of the Balanced Minimum Evolution Method of Phylogenetic Inference and Its Relationship to Weighted LeastSquares Tree Fitting. J. of Mol. Biol. Evol. 2004 21(3): 587-598.

2. R. Desper, R. Gascuel. Fast and accurate phylogeny reconstruction algorithms based on the minimum evolution principle. J. Comp. Biol. 2002 9: 687-705.

3. Y. Pauplin. Direct calculation of a tree length using a distance matrix. J Mol Evol. 2000 51(1): 41-7.

4. N. Saitou, M. Nei. The Neighbor-Joining Method: a New Method for Reconstructing Phylogenetic Trees. Mol Biol Evol. 1987 4(4): 406-25.

5. O. Gascuel. BIONJ: an Improved Version of the NJ Algorithm Based on a Simple Model of Sequence Data. Mol Biol Evol. 1997 14(7):685-95.

6. Bruno WJ, Socci ND, Halpern AL. Weighted Neighbor Joining: a LikelihoodBased Approach to Distance-Based Phylogeny Reconstruction. Mol Biol Evol. 2000 17(1):189-97.

7. K. Atteson. The Performance of Neighbor-Joining Algorithms of Phylogeny Reconstruction. Proceedings of the Third Annual International Conference on Computing and Combinatorics, Lecture Notes In Computer Science Vol. 12761997 :101 - 110.

8. W. Day, D. Sankoff. Computational Complexity of Inferring Phylogenies by Compatibility. Systematic Zoology,1986 35(2):224-229.

9. J. Felsenstein. An Alternating Least Squares Approach to Inferring Phylogenies from Pairwise Distances Systematic Biology,1997 46(1):101-111.

10. D. Benedetto, E. Caglioti and V. Loreto. Language Trees and Zipping. Physical Review Letters 2002 88(4):048702-1-04872-4.

11. R. Cilibrasi, P. Vitányi. Clustering by Compression. IEEE Transactions on Information Theory, 2005 51(4):1523-1545.

12. T. Shigezumi. Robustness of Greedy Type Minimum Evolution Algorithms. Dept. of Math. and Comp. Sciences Tokyo Institute of Technology Research Reports (Series C), C-218, 2005. http://www .is.titech.ac.jp/research/research-report/ C/C-218.ps.gz 